\title{
Enhanced oxygen diffusion in nano-structured ceria
}

\author{
Yu. I. Boyko $^{1}$ - V. I. Biletskyi ${ }^{1}$-V. V. Bogdanov ${ }^{1} \cdot$ R. V. Vovk ${ }^{1}$ - G. Ya. Khadzhai ${ }^{1}$ - I. L. Goulatis ${ }^{1}$ - A. Chroneos ${ }^{2,3}$
}

Received: 30 October 2017 / Accepted: 15 December 2017 / Published online: 15 January 2018

(c) The Author(s) 2018. This article is an open access publication

\begin{abstract}
Nano-structured polycrystalline $\mathrm{CeO}_{2}$ is proposed to be used as a candidate electrolyte material for solid oxide fuel cells (SOFC) operating at temperatures $\leq 600^{\circ} \mathrm{C}$. Here we show that in this material the diffusion activation energy of oxygen ions is $0.35 \mathrm{eV}$. The diffusion mechanism corresponds to the "single-file" diffusion mechanism, associated with the presence of one-dimensional channels in the anion sublattice of $\mathrm{CeO}_{2}$. The small grain size of the material $\approx 10 \mathrm{~nm}$, as well as its stoichiometric state $\left(\mathrm{Ce}_{2} \mathrm{O}_{3}\right)$, minimize the contribution of the electronic and polaron components of the electrical conductivity of this material, which also contribute to the optimization of SOFC operation.
\end{abstract}

\section{Introduction}

SOFCs are characterized by increased energy efficiency (>60\%), simplicity of technical realization, and limited emissions during operation [1-10]. These constitute SOFC important for both stationary and mobile applications. The efficiency of SOFC is strongly dependent upon the diffusion of oxygen ions $\left(\mathrm{O}^{2-}\right)$ in the electrolyte. Presently, the most widely used electrolyte is the $\mathrm{ZrO}_{2}$ polycrystalline doped with oxides such as $\mathrm{Y}_{2} \mathrm{O}_{3}$ (to form YSZ). These dopants in an amount of $\approx 10 \mathrm{~mol} \%$ are increasing the concentration of vacancies in the oxygen sublattice of the electrolyte material, which in turn facilitate oxygen ions diffusion. Additionally, yttria stabilizes the fluorite crystal structure of $\mathrm{ZrO}_{2}$ from room temperature to $1000{ }^{\circ} \mathrm{C}$ [1].

The power of the SOFC source is determined by the flux $\mathrm{J}$ of $\mathrm{O}^{2-}$ ions diffusing through the electrolyte from the cathode to the anode at a partial oxygen pressure of $\approx 0.2$ bar. The value of the oxygen ions flux $\mathrm{J}$ is proportional to the electrical conductivity coefficient of the electrolyte material $\sigma$, which in turn depends from the diffusion coefficient $D_{V}$ and the temperature $\mathrm{T}$ :

\section{A. Chroneos}

ab8104@coventry.ac.uk

1 Physics Department, V. Karazin Kharkiv National University, Svobody Sq. 4, Kharkiv 61022, Ukraine

2 Faculty of Engineering, Environment and Computing, Coventry University, Priory Street, Coventry CV1 5FB, UK

3 Department of Materials, Imperial College London, South Kensington Campus, London SW7 2AZ, UK
$\mathrm{J} \sim \sigma \sim \mathrm{D}_{\mathrm{V}} \sim \exp (-\mathrm{Q} / \mathrm{kT})$.

Here, $D_{V}$ is the diffusion coefficient of oxygen ions $\mathrm{O}^{2-}$ in the electrolyte, $\mathrm{Q}$ is the activation energy of diffusion, and $\mathrm{k}$ is Boltzmann's constant. The really consumed power of SOFC using electrolyte $\mathrm{YSZ}\left(\mathrm{ZrO}_{2}+8 \% \mathrm{Y}_{2} \mathrm{O}_{3}\right)$ is determined by the value of $\sigma \approx\left(10^{-3}-10^{-1}\right) \Omega^{-1} \mathrm{~cm}^{-1}$, which is achieved in the temperature range $\mathrm{T} \approx 600-1000{ }^{\circ} \mathrm{C}$. The activation energy for $\mathrm{YSZ}$ is $0.96 \mathrm{eV}$ [1]. The high operating temperature of the SOFC source with this electrolyte significantly complicates its technical implementation. Additionally, prolonged operation at high temperature is accompanied by segregation of the dopant atoms leading to the formation of microscopic emissions within the grain boundaries of the electrolyte substance. This causes an increase in the electrical resistance of the electrolyte and, correspondingly, a decrease in the power of the device.

Therefore, there is technological requirement to introduce materials for the SOFC electrolyte that will (i) have high oxygen diffusion (i.e. lower activation energy of diffusion) and (ii) minimum segregation of impurities. Segregation can be limited by using an electrolyte with a minimum content of impurities, and also by lowering the operating temperature. $\mathrm{CeO}_{2}$ is considered as an intermediate-temperature SOFC (IT-SOFC) electrolyte as it has an electric conductivity coefficient $\sigma$ in the temperature range $500-1000{ }^{\circ} \mathrm{C}$, which is an order of magnitude larger than YSZ [10-12]. Here we propose to use nano-structured polycrystalline $\mathrm{CeO}_{2}$ as an IT-SOFC electrolyte because it satisfies these requirements.

Nevertheless, as it is shown in experimental studies, the commonly used coarse-grained $(\geq 100 \mathrm{~nm})$ cerium dioxide to a large extent deviates from the stoichiometric 
composition in this temperature range, especially under conditions of even a small restorative atmosphere. Such a property of the usual size scale $\mathrm{CeO}_{2}$ crystals leads to the fact that electrons and small-radius polarons (SRP) make an important contribution to the electrical conductivity of this substance. The latter are formed as a result of the interaction of the $4 \mathrm{f}$ electrons of $\mathrm{Ce}^{3+}$ ions with longitudinal optical vibrations of the crystal lattice [13]. The appearance of $\mathrm{Ce}^{3+}$ ions in ceria is due to the changes in its stoichiometric composition (in its chemical formula, $\mathrm{CeO}_{2-\mathrm{x}}, \mathrm{x}>0$ ). Naturally, the participation of electrons and polarons in the conductivity of ceria adversely affects its use as an electrolyte for SOFC, since the optimal electrolyte for SOFC must have a sufficiently high conductivity exclusively in relation to oxygen ions.

Here we propose to use stoichiometric cerium dioxide as the contribution of electrons and SRP to the electrical conductivity of this substance is minimal, and the ionic conductivity in the oxygen sublattice is fully realized even in a weakly restorative environment (i.e. air atmosphere). The results indicate that polycrystalline nano structured $\mathrm{CeO}_{2}$ with a grain size of $\approx(10-20) \mathrm{nm}$ is characterized by more than two orders larger conductivity coefficient $\sigma$ in comparison to the conductivity of a conventional polycrystal with a grain size $\approx(50-100) \mathrm{nm}$.

This effect is due to the increase in the oxygen diffusion because of the transport of oxygen ions along the grain boundaries, as well as along the one-dimensional channels formed in the oxygen sublattice of small (nano-sized) crystalline grains. Both diffusion mechanisms are characterized by a much lower activation energy than the activation energy of bulk diffusion in the grains. This motivates the use of this material as an electrolyte for SOFC, operating at temperatures $\approx(300-600){ }^{\circ} \mathrm{C}$, without significant loss of power as compared to SOFC with the YSZ electrolyte. Naturally, a decrease in the temperature range should also help to reduce the "aging" effect of the source, i.e., its degradation during long-term use.

\section{Experimental methodology}

The studied $\mathrm{CeO}_{2}$ crystals were synthesized using the "sol-gel" chemical method, in which it is possible to control the size of the crystals by varying the molar ratio of $\mathrm{H}_{2} \mathrm{O}$ /surfactant [14]. Crystals of two sizes were used: macro-crystals with a size of $\approx(50-100) \mathrm{nm}$, and nanocrystals $\approx(10-20) \mathrm{nm}$. Nano-dimensional crystals were characterized by a special structural state in connection with the manifestation of the so-called "size effect". Immediately after the preparation, all the crystals were subjected to a stabilizing heat treatment by annealing in an air atmosphere at a temperature of $1000{ }^{\circ} \mathrm{C}$ for $2 \mathrm{~h}$. The average crystal size was determined by electron microscopy.

To measure the electrical conductivity, cylindrical samples with a diameter of $4 \mathrm{~mm}$ and a height of $1.5 \mathrm{~mm}$ were prepared. Each sample was "compacted" in a special mold at room temperature. The porosity of sample $\theta$ was calculated by comparing the sample's density with the tabulated value of the $\mathrm{CeO}_{2}$ crystal density. In the following, the porosity value was taken into account to correct the measured value of the electrical conductivity coefficient of the material [15]. In all the samples we studied, $\theta \ll 1$ and, consequently, had practically no effect on the value of the measured specific electrical conductivity of the samples.

To measure the value of the electrical conductivity coefficient $\sigma$, the sample was placed in a cell, the body of which was made of an insulator (pyrophyllite), whereas as contacts we used stainless steel rods. The sample temperature during the conductivity measurements was monitored with a copper-constantan thermocouple and varied in the interval (100-600) ${ }^{\circ} \mathrm{C}$. The measurement of $\sigma$ was carried out by a standard method using an alternating current with frequency $\approx 1 \mathrm{kHz}$ in air atmosphere.

\section{Results and discussion}

\subsection{Structural state and stoichiometry of $\mathrm{CeO}_{2}$ nano-crystals}

The ratio of the radii of the ions $\mathrm{Ce}^{4+}$ and $\mathrm{O}^{2-}$ is 0.77 , causing the formation of the "fluorite" type lattice [16]. In this lattice $\mathrm{Ce}^{4+}$ form a cubic face-centered lattice, while $\mathrm{O}^{2-}$ are located in the centers of small cubes, into which the unit cell can be divided. Therefore, each cerium ion is surrounded by eight oxygen ions located at the vertices of the cube, and each oxygen ion is surrounded by four cerium ions located at the vertices of the regular tetrahedron. Such a crystal structure corresponds to the densest packing of cerium and oxygen ions in the (111) planes with coordination (8:4) and at the same time ensures the fulfillment of the electrical neutrality condition of the crystal.

However, the structural state of the $\mathrm{CeO}_{2}$ crystal described above is characteristic only for the crystals of the usual size scale $\geq 100 \mathrm{~nm}$. With a decrease in crystal size to $\approx 10 \mathrm{~nm}$ (nano-crystal), a so-called near-surface structural rearrangement occurs [17]. The reason for such a rearrangement is that for ions located at the surface of the crystal and in a near-surface layer of thickness $\delta \approx 3 a$ ( $a$ is the parameter of the crystal lattice), the number of the nearest neighbors (the number of ions of the first and subsequent coordination spheres) decreases.

An elementary estimation shows that for a crystal size $\mathrm{d} \approx 10 \mathrm{~nm}$, about half of ions are "sensing" the absence of 
the half of the space. The natural consequence of the change in the number of nearest neighbors (change in the coordination number) in nano-crystals is that the "surface" and "nearsurface" ions are forced to shift to new positions. Thus, the frequencies of ion oscillations change, their polarization changes and, accordingly, the character of their interaction changes. In ionic compounds of the $\mathrm{AB}_{2}$ type, the emerging polarization, although significant, is still insufficient for a transition from the "fluorite" structure to some other structure with a different coordination number.

As a rule, there form specific transition structures-the so-called "layered" crystal lattices [16]. As a result of this transformation, symmetry and translational order in the ionic arrangement in the crystals of this class are violated, which ultimately causes a change in the valency of the cations and, correspondingly, a change in the stoichiometry. It is this structural adjustment, i.e. the formation of a "layered" crystal lattice, as well as a change in the stoichiometric composition, occur in $\mathrm{CeO}_{2}$ crystals with a decrease in their size to $\approx 10 \mathrm{~nm}$. This is supported by the following experimental facts. Firstly, spectral studies have shown that in crystals of this size, the valence of the cation changes, $\mathrm{Ce}^{4+} \rightarrow \mathrm{Ce}^{3+}$, i.e., there is a transformation of $\mathrm{CeO}_{2}$ to the sesquioxide $\mathrm{Ce}_{2} \mathrm{O}_{3}$ [18]. Secondly, due to a change in the valence of the cation, additional ("nonstoichiometric") vacancies are formed in the anion sublattice of the crystal. Indeed, from the condition of the electric neutrality of the crystal it follows that in the transformation of two $\mathrm{Ce}^{4+}$ ions into $\mathrm{Ce}^{3+}$ ions, one additional oxygen vacancy forms. Thus, it should be considered that the "layered" lattice in ionic $\mathrm{AB}_{2}$ type crystals is formed by imposing a series of packets, each of which consists of a layer of cations enclosed between two layers of polarized anions [16].

In the case of $\mathrm{CeO}_{2}$ nano-crystals, during the formation of a "layered" crystal lattice, each packet is formed from a layer of $\mathrm{Ce}^{3+}$ ions (cations) located between two layers of $\mathrm{O}^{2-}$ (anions) ions. One such layer is schematically represented in Fig. 1. A peculiarity of this structure is the asymmetric coordination of ions: while the three cations are symmetrically surrounded by six anions located at the apexes of the octahedron, two anions, located in the layer and connected with one cation on one side of it. The polarization of ions due to this structure practically does not change the distance between oppositely charged ions, but the distance between ions of the same sign substantially increases. To preserve the greatest degree of ions' packing in the (111) planes, rows are formed in them in which ions of the same sign are located. Due to the decrease in the bond energy between the ions in these rows, the formation of "nonstoichiometric" vacancies becomes more likely particularly in the oxygen sublattice. This conclusion was confirmed experimentally [19], in which it was determined that the energy of vacancy formation in the

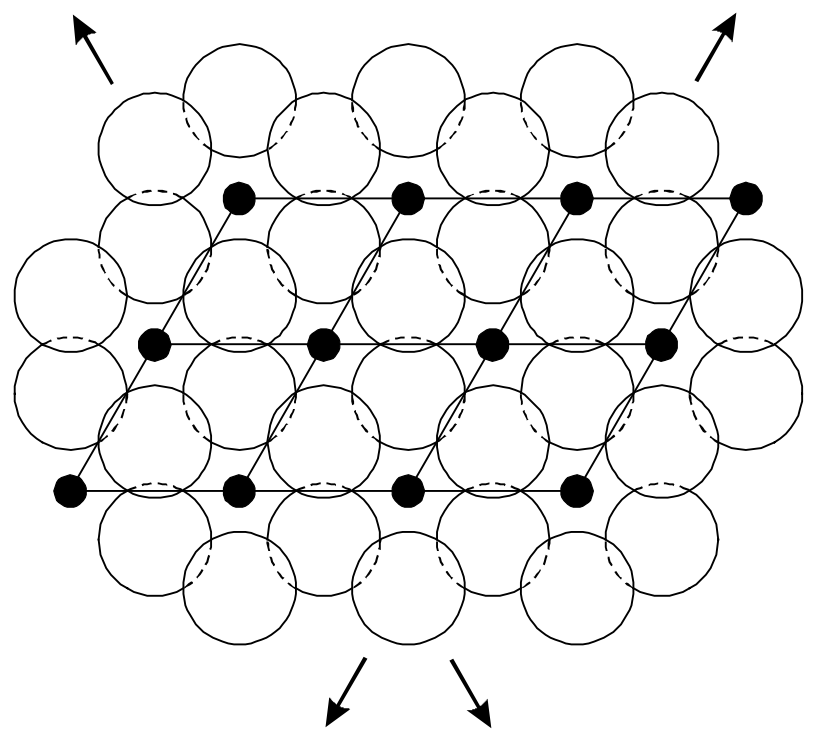

Fig. 1 The crystal structure of a layered lattice. filled circle-Ce; open circle- $\mathrm{O}$

oxygen sublattice of the $\mathrm{CeO}_{2}$ crystal decreases from $4.5 \mathrm{eV}$ (in the bulk crystal with the "fluorite" lattice) to $0.25 \mathrm{eV}$ (nano-crystal, "layered" lattice).

The process of structural rearrangement occurring in $\mathrm{CeO}_{2}$ nano-crystals, leads to the formation of ordered clusters (one-dimensional channels) consisting of "nonstoichiometric" oxygen vacancies. Experimentally, the formation of such one-dimensional hollow channels in $\mathrm{CeO}_{2}$ nanocrystals was detected by high-resolution electron microscopy [20].

To summarize, the structural rearrangement occurring in $\mathrm{CeO}_{2}$ crystals with a decrease in its size to a value of about $10 \mathrm{~nm}$ causes the transformation of the "fluorite" type crystalline lattice into a "layered" type lattice. Such a rearrangement is associated with a "dimensional effect" and is accompanied by a change in the stoichiometry of the crystal, i.e. it is accompanied by a phase transformation $\mathrm{CeO}_{2} \rightarrow \mathrm{Ce}_{2} \mathrm{O}_{3}$ with simultaneous formation of one-dimensional hollow channels consisting of oxygen vacancies in the anion sublattice of the crystal. Such channels are located in close-packed crystallographic planes (111), oriented along [111] type directions, and can penetrate crystals with a size $\leq 10 \mathrm{~nm}$.

The presence of one-dimensional hollow channels in the anion sublattice of $\mathrm{CeO}_{2}$ nano crystals causes the accelerated diffusion transport of oxygen ions due to a decrease in the activation energy (the so-called "single-file diffusion" mechanism) [21, 22]. It is this unique structure of nanometer-sized crystals that motivates their use as electrolytes in SOFCs. Additionally, the use of the $\mathrm{CeO}_{2}$ electrolyte in the form of a nano-structured material with grain size $\approx 10 \mathrm{~nm}$ can also contribute to an increase in the oxygen self-diffusion due to their migration along the grain boundaries. 
In a polycrystal with such a small grain size, the total area of the boundaries increases significantly, and the diffusion transport of ions along the boundaries is much faster than in the grain volume, since $D_{B} \gg D_{V}\left(D_{B}\right.$ is the diffusion coefficient in the grain boundary) [23]. A quantitative assessment of the contribution of the two possible ways to enhance the diffusion permeability of oxygen ions in nanostructured polycrystals, follows in the next section.

Notably, in the nano-structured material $\mathrm{CeO}_{2}$ in the equilibrium state is practically converted to the sesquioxide $\mathrm{Ce}_{2} \mathrm{O}_{3}$, i.e., the stoichiometry of this substance changes more. This blocks the formation of polarons, and the small grain size significantly limits the mean free path of the electrons. Thus, the contribution of these undesirable charge carriers to the conductivity of nano-structured $\mathrm{CeO}_{2}$ is minimized, which also reinforces the use of cerium oxide as an electrolyte in IT-SOFC.

\subsection{Intensification of oxygen diffusion in nano-structured $\mathrm{CeO}_{2}$}

This section considers the quantitative evaluation of the degree of intensification of oxygen diffusion in the nanostructured polycrystalline $\mathrm{CeO}_{2}$. Firstly, we will estimate the possible contribution in the process of enhancing of the oxygen diffusion in a nano-structured polycrystal due to the increase of the total area of grain boundaries. The transport of a substance in a polycrystalline material taking into account the presence of grain boundaries is characterized by an effective diffusion coefficient

$\mathrm{D}_{\mathrm{ef}}=\mathrm{C}_{1} \mathrm{D}_{\mathrm{V}}+\mathrm{C}_{2} \mathrm{D}_{\mathrm{B}} \delta / \mathrm{d}$,

where $D_{V}$ and $D_{B}$ are respectively the diffusion coefficients in the grain volume and in the boundary, $\delta$ is the width of the boundary, $\mathrm{d}$ is the grain size, $\mathrm{C}_{1}$ and $\mathrm{C}_{2}$ are numerical factors [23]. For the quantitative estimation of the possible increase in the oxygen diffusion due to the presence of grain boundaries, we can use the dimensionless ratio: $\chi=D_{\mathrm{ef}} / \mathrm{D}_{\mathrm{V}}$. Herewith, it should be taken into account that at temperatures $<500{ }^{\circ} \mathrm{C}$ the main process that determines the diffusion transport of matter is the movement of oxygen along the grain boundaries, that is, the first term in Eq. 2 can be neglected, and thus:

$\chi \approx \mathrm{C}_{2} \mathrm{D}_{\mathrm{B}} \delta / \mathrm{dD}_{\mathrm{V}}$.

Substituting in Eq. 3 reasonable values of: $\mathrm{C} 2 \approx 1$, $\delta \approx 1 \mathrm{~nm}, \mathrm{D}_{\mathrm{B}} / \mathrm{D}_{\mathrm{V}} \approx 10^{4}$ (at $\mathrm{T} \approx 500{ }^{\circ} \mathrm{C}$ ), $\mathrm{d} \approx 10 \mathrm{~nm}$, we get: $\chi \approx 10^{3}$. The evaluation shows that using the nano-structured polycrystalline $\mathrm{CeO}_{2}$ as an electrolyte for SOFC, it is possible to substantially increase the effective diffusion coefficient of oxygen ions, and, consequently, the electrical conductivity of this material at temperatures $\leq 500{ }^{\circ} \mathrm{C}$. Next, we estimate the possible increase in the oxygen diffusion in $\mathrm{CeO}_{2}$ nano-crystals in which there exist one-dimensional hollow channels in the anion sublattice. As already indicated, in this case, the transport of oxygen ions can be realized by the single-file diffusion mechanism [21, 22]. This mechanism differs significantly from the classical diffusion mechanism, both, within the volume of the material and along the grain boundaries. The difference is as follows: the classical particle's diffusion mechanism in crystals is based on the model of "random walks" [23].

According to this model, the jumps of particles in the process of their thermal migration occur independently of each other, that is, there is no correlation between them: the succeeding jump of the particle does not depend on what its previous jump was. With such a diffusion mechanism, the radius-vector of the mean displacement of a large number of particles is zero, but the mean-square displacement is different from zero and is described by the Einstein-Smoluchowski relation: $\left\langle\mathrm{X}^{2}(\mathrm{t})\right\rangle=2 \mathrm{D}_{\mathrm{V}} \mathrm{t}$ ( $\mathrm{t}$ is the diffusion time). The correctness of this conclusion was confirmed by previous experimental and theoretical studies [23].

Notably, under the action of this mechanism, the average value of the diffusion displacement of the particle from its initial position $\mathrm{L}_{\mathrm{d}}=\left(2 \mathrm{D}_{\mathrm{V}} \mathrm{t}\right)^{1 / 2}$ is much less than the total distance $\mathrm{L}$ that it travels during this time, i.e. $\mathrm{L}_{\mathrm{d}} \ll \mathrm{L}$. This highlights the low efficiency of matter's transport by the classical diffusion mechanism in the volume of the crystal and explains why the penetration depth of the diffusing particles is so insignificant even at a high temperature and for a sufficiently long annealing times.

In the case of "single-file diffusion", the thermal migration of the moving particles is limited to a one-dimensional hollow channel, along which the particles can move only in one direction, and during the migration process cannot pass each other. The root-mean-square displacement of the particles in this diffusion mechanism is described by the following relation:

$\left\langle\mathrm{X}^{2}(\mathrm{t})\right\rangle=2 \mathrm{Ft}^{1 / 2}$.

Here $\mathrm{F}$ is mobility, a specific parameter characterizing the displacement of diffusing particles in the "single-file" diffusion regime [21]. By itself, the parameter F cannot be used to characterize the efficiency of diffusion. However, if we apply the model of "random walks" to one particle, moving in one direction and not experiencing interaction with other particles (the analog of "single-file diffusion"), then the mean square displacement can approximately be described with the help of this relation:

$\left\langle\mathrm{X}^{2}(\mathrm{t})\right\rangle \approx \lambda\langle\mathrm{X}(\mathrm{t})\rangle$.

Here $\langle\mathrm{X}(\mathrm{t})\rangle$ is the average displacement of the moving particle in the one-dimensional channel and $\lambda$ is the 
distance between the moving particles. Assuming that, in analogy with the classical diffusion mechanism, as applied for the "single-file diffusion" the relation describing the average displacement in the form: $\langle\mathrm{X}(\mathrm{t})\rangle=\left(2 \mathrm{D}_{\mathrm{sf}} \mathrm{t}\right)^{1 / 2}$ is also satisfied and, taking into account Eq. 5: $\mathrm{D}_{\mathrm{sf}} \approx 2 \mathrm{~F}^{2} / \lambda^{2}$. In this relation, the parameter $\mathrm{D}_{\mathrm{sf}}$ has the dimension of the diffusion coefficient and can characterize the efficiency of the ions' motion by the "single-file diffusion" mechanism. As before, to give a quantitative estimation of the possible degree of change in the efficiency of matter's transport by this mechanism, in comparison to the classical diffusion mechanism acting in the volume of the crystal, we form the dimensionless ratio:

$\chi=\mathrm{D}_{\mathrm{sf}} / \mathrm{D}_{\mathrm{V}} \approx 2 \mathrm{~F}^{2} / \lambda^{2} \mathrm{D}_{\mathrm{V}}$.

Substituting in Eq. 6 reasonable values: $\lambda \approx 1 \mathrm{~nm}$, $\mathrm{D}_{\mathrm{V}} \approx 10^{-11} \mathrm{~m}^{2} \mathrm{~s}^{-1}$ (ionic crystals at temperature $\mathrm{T} \approx 500^{\circ} \mathrm{C}$ [23]), as well as the mobility value characteristic for nanodimensional objects $\mathrm{F} \approx 10^{-13} \mathrm{~m}^{2} \mathrm{~s}^{-1 / 2}$ [21], we obtain: $\chi \approx 10^{3}$. Thus, using an electrolyte based on $\mathrm{a} \mathrm{CeO}_{2}$ nanostructured polycrystalline with a grain size of $\approx 10 \mathrm{~nm}$, it is possible to increase oxygen diffusion by three orders of magnitude, and thus to lower the operating temperature of the source to $\leq 600{ }^{\circ} \mathrm{C}$.

\subsection{Experimental results}

The results of the measurements demonstrated in Figs. 2 and 3 , show that the specific electric conductivity for the both types of samples, depends essentially on the temperature and increases exponentially by its increase. The conductivity of the nano-structured sample at a temperature of $\approx 300{ }^{\circ} \mathrm{C}$ is more than two orders of magnitude higher than the electrical conductivity of the coarse-grained sample (see Fig. 2). A

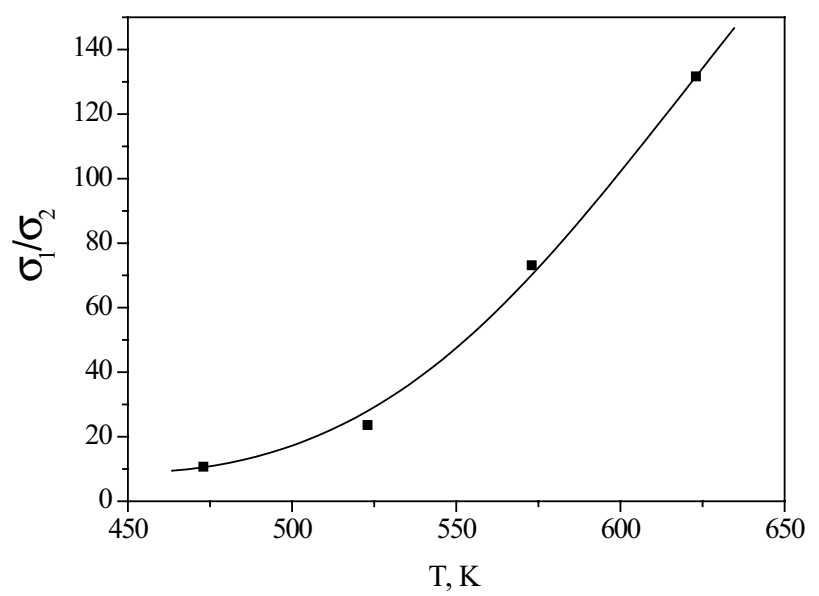

Fig. 2 The temperature dependence of the electrical conductivities ratio of the nano-structured $\left(\sigma_{1}\right)$ and the coarse-grained $\left(\sigma_{2}\right)$ polycrystals

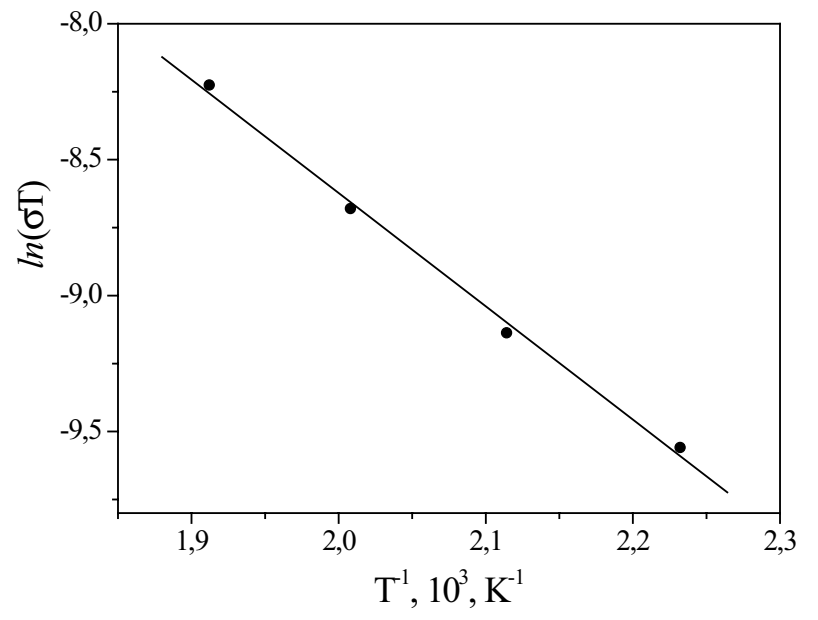

Fig. 3 Temperature dependence of the electrical conductivity of the $\mathrm{CeO}_{2}$ nano-structured polycrystals

specific role in this may played the specific scattering mechanisms, caused by the existents of structural and kinematic anisotropy in the system [24-26]. The processing of the experimental data in the coordinates $\ln (\sigma \mathrm{T})-1 / \mathrm{T}$ indicates a significant decrease in the value of the activation energy $\mathrm{Q}$ from $\approx 0.87 \mathrm{eV}$ for the coarse-grained material to $\approx 0.35 \mathrm{eV}$ for the nano-structured polycrystal (refer to Fig. 3). This small value of the activation energy in the case of a nanostructured material is comparable to the energy of formation of oxygen vacancies $(\approx 0.25 \mathrm{eV})$ in $\mathrm{CeO}_{2}$ nano-crystals, when there are one-dimensional hollow channels present that are formed by the vacancies in the oxygen sublattice [18].

\section{Conclusions}

It can be concluded that $\mathrm{CeO}_{2}$ in a nano-structured polycrystalline form is very promising for its use as an electrolyte for IT-SOFC. The experimental data indicates that the most probable factor ensuring an increase in the efficiency of oxygen diffusion is the presence of one-dimensional channels formed by vacancies in the anion sublattice. In this case, in our opinion, the "single-file" diffusion mechanism is realized, that is characterized by a low value of the activation energy of diffusion $\approx 0.35 \mathrm{eV}$. This constitutes nano-structured polycrystalline $\mathrm{CeO}_{2}$ a better candidate electrolyte material for IT-SOFC as compared to coarse grained $\mathrm{CeO}_{2}$ or traditional materials such as YSZ.

Open Access This article is distributed under the terms of the Creative Commons Attribution 4.0 International License (http://creativecommons.org/licenses/by/4.0/), which permits unrestricted use, distribution, and reproduction in any medium, provided you give appropriate credit to the original author(s) and the source, provide a link to the Creative Commons license, and indicate if changes were made. 


\section{References}

1. N. Minh, J. Am. Ceram. Soc. 76, 563 (1993)

2. S.C. Singhal, Solid State Ionics. 135, 305 (2000)

3. B.C. Steele, A. Heinzel, Nature 414, 345 (2001)

4. J. Fleig, Annu. Rev. Mater. Res. 33, 361 (2003)

5. A.J. Jacobson, Chem. Mater 22, 660 (2010)

6. J.B. Goodenough, Annu. Rev. Mater. Res. 33, 91 (2003)

7. A. Tarancón, M. Burriel, J. Santiso, S.J. Skinner, J.A. Kilner, J. Mater. Chem. 20, 3799 (2010)

8. I.D. Seymour, A. Chroneos, J.A. Kilner, R.W. Grimes, Phys. Chem. Chem. Phys. 13, 15305 (2011)

9. I.D. Seymour, A. Tarancón, A. Chroneos, D. Parfitt, J.A. Kilner, R.W. Grimes, Solid State Ionics 216, 41 (2012)

10. M.J.D. Rushton, A. Chroneos, Sci. Rep. 4, 6068 (2014)

11. R. Blumenthal, F. Brugner, J. Garmier, J. Electrochem. Soc. 120, $1230(1973)$

12. H. Tuller, A. Nowick, J. Electrochem. Soc. 122, 255 (1975)

13. V. Ogorodnik, G. Galina, G. Kalnaya, R. Priscepa, Vestnik NTUU, 30, 82

14. A. Serra, V. Severino, P. Calefi, S. Cicilini, J. Alloys Compd. 323, $667(2001)$

15. S. David, M. Blaszkiewicz, R. Newnham, J. Am. Ceram. Soc. 73, $2187(1990)$
16. R. Evans, Vvedenie v kristallohimiyu (Goshimizdat, Moskva, 1948), p. 367

17. Yu Petrov, Fizika malyh chastitc (Nauka, Moskva, 1982), p. 275

18. V. Seminko, P. Maksimchuk, N. Kononets, E. Okrushko, I. Bespalova, A. Masalov, Yu. Malykin, Yu. Boyko, Visnyk HNU, «Fizika» 24, 20 (2016)

19. N. Skorodumova, S. Simak, B. Lundqvist, I. Abrikosov, B. Johansson, Phys. Rev. Lett. 89, 601 (2002)

20. F. Esch, S. Fabris, L. Zhou, T. Montini, C. Africh, P. Fomasiero, G. Comelli, R. Rosei, Science 309, 752 (2005)

21. K. Hahn, J. Karger, V. Kukla, Singl, Phys. Rev. Lett. 76, 2762 (1996)

22. S. Nedea, Phys. Rev. E 67, 1 (2003)

23. B. Bokshtein, S. Bokshtein, A. Zhukhovitskij, Termodinamika $i$ kinetika diffuzii v tverdyh telah (Metallurgiya, Moskva, 1974), p. 280

24. P.G. Curran, V.V. Khotkevych, S.J. Bending, A.S. Gibbs, S.L. Lee, A.P. Mackenzie, Phys. Rev. B 84, 104507 (2011)

25. V.M. Apalkov, M.E. Portnoi, Phys. Rev. B 65, 125310 (2002)

26. I.N. Adamenko, K. E.Nemchenko, V.I. Tsyganok, A.I. Chervanev, Low Temp. Phys. 20, 498 (1994) 